\title{
THE VLT LEGA-C SPECTROSCOPIC SURVEY: THE PHYSICS OF GALAXIES AT A LOOKBACK TIME OF 7 Gyr
}

\author{
A. van der Wel ${ }^{1}$, K. Noeske ${ }^{1}$, R. Bezanson ${ }^{2}$, C. Pacifici ${ }^{3}$, A. Gallazzi ${ }^{4}$, M. Franx ${ }^{5}$, J. C. Muñoz-Mateos $^{6}$, E. F. Bell ${ }^{7}$, \\ G. Brammer ${ }^{8}$, S. Charlot ${ }^{9}$, P. Chauké ${ }^{1}$, I. Labbé ${ }^{5}$, M. V. Maseda ${ }^{5}$, A. Muzzin ${ }^{10}$, H.-W. Rix ${ }^{1}$, D. Sobral ${ }^{5,11}$, \\ J. VAN DE SANDE ${ }^{12}$, P. G. VAN DOKKUM ${ }^{13}$, V. Wild ${ }^{14}$, AND C. Wolf ${ }^{15}$ \\ ${ }^{1}$ Max-Planck Institut für Astronomie, Königstuhl 17, D-69117, Heidelberg, Germany; vdwel@mpia.de \\ ${ }^{2}$ Steward Observatory, University of Arizona, 933 North Cherry Avenue, Tucson, AZ 85721, USA \\ ${ }^{3}$ Astrophysics Science Division, Goddard Space Flight Center, Code 665, Greenbelt, MD 20771, USA \\ ${ }^{4}$ INAF-Osservatorio Astrofisico di Arcetri, Largo Enrico Fermi 5, I-50125 Firenze, Italy \\ ${ }^{5}$ Leiden Observatory, Leiden University, P.O.Box 9513, NL-2300 AA Leiden, The Netherlands \\ ${ }^{6}$ European Southern Observatory, Alonso de Crdova 3107, Casilla 19001, Vitacura, Santiago, Chile \\ ${ }^{7}$ Department of Astronomy, University of Michigan, 1085 S. University Ave, Ann Arbor, MI 48109, USA \\ ${ }^{8}$ Space Telescope Science Institute, 3700 San Martin Drive, Baltimore, MD 21218, USA \\ 9 UPMC-CNRS, UMR 7095, Institut d'Astrophysique de Paris, F-75014 Paris, France \\ ${ }^{10}$ Institute of Astronomy, University of Cambridge, Madingley Road, Cambridge, CB3 0HA, UK \\ ${ }_{11}$ Department of Physics, Lancaster University, Lancaster, LA1 4 YB, UK \\ ${ }^{12}$ Sydney Institute for Astronomy, School of Physics, University of Sydney, NSW 2006, Australia \\ ${ }^{13}$ Department of Astronomy, Yale University, New Haven, CT 06511, USA \\ ${ }^{14}$ School of Physics and Astronomy, University of St Andrews, North Haugh, St Andrews, KY16 9SS, UK \\ ${ }^{15}$ Research School of Astronomy and Astrophysics, Australian National University, Canberra, ACT 2611, Australia \\ Received 2016 February 12; accepted 2016 March 17; published 2016 April 22
}

\begin{abstract}
The Large Early Galaxy Census (LEGA-C ${ }^{16}$ ) is a Public Spectroscopic Survey of $3200 K$-band selected galaxies at redshifts $z=0.6-1.0$ with stellar masses $M_{*}>10^{10} M_{\odot}$, conducted with VIMOS on ESO's Very Large Telescope. The survey is embedded in the COSMOS field (R.A. $=10 \mathrm{~h} 00$; decl. $=+2 \mathrm{deg}$ ). The $20 \mathrm{hr}$ long integrations produce high-signal-to-noise ratio continuum spectra that reveal ages, metallicities and velocity dispersions of the stellar populations. LEGA-C's unique combination of sample size and depth will enable us for the first time to map the stellar content at large lookback time, across galaxies of different types and star formation activity. Observations started in 2014 December and are planned to be completed by mid 2018, with early data releases of the spectra and value-added products. In this paper we present the science case, the observing strategy, an overview of the data reduction process and data products, and a first look at the relationship between galaxy structure and spectral properties, as it existed 7 Gyr ago.
\end{abstract}

Key words: galaxies: evolution - galaxies: fundamental parameters - galaxies: general - galaxies: stellar content surveys

\section{INTRODUCTION}

Our understanding of galaxy evolution at late cosmic epochs has been revolutionized by spectra from the Sloan Digital Sky Survey (York et al. 2000): their high signal-to-noise and high resolution have allowed accurate measurements of the (lightweighted) integrated stellar ages and metallicities of galaxies (e.g., Kauffmann et al. 2003b; Gallazzi et al. 2005). These data have brought into clear focus the multi-variate correlations between stellar population properties and mass (Kauffmann et al. 2003c), structure (e.g., concentration, bulge-to-disk ratio; Kauffmann et al. 2003c), size (van der Wel et al. 2009), stellar velocity dispersion (Gallazzi et al. 2006; Graves et al. 2009), nuclear activity (Kauffmann et al. 2003a; Schawinski et al. 2009), and environment (Kauffmann et al. 2004; Pasquali et al. 2010; Thomas et al. 2010), which have greatly illuminated the processes that drive star formation and the ongoing assembly of present-day galaxies. The main limitation of examining present-day galaxies for the purpose of reconstructing their formation history is that most of the star formation occurred in the distant past: mean stellar ages of $L^{*}$ galaxies are typically well over $5 \mathrm{Gyr}$ and it is difficult to resolve star formation histories from integrated spectra.

\footnotetext{
${ }^{16}$ http://www.mpia.de/home/legac/index.html
}

Now LEGA-C is obtaining spectra of similar quality for $\sim 3200 \mathrm{~K}$-band selected galaxies in the redshift range $0.6<z<1.0$, at a lookback time of $6-8$ Gyr. LEGA-C is a 4 year, 128-night Public Spectroscopic Survey with VLT/ VIMOS (Le Fèvre et al. 2003), one of the most extensive extragalactic surveys on an 8-10 m class telescope, and is unique in its combination of depth and resolution. We know that $~ 50 \%$ of all stars in the universe formed since $z \sim 1$ (e.g., Dickinson et al. 2003; Rudnick et al. 2003; Ilbert et al. 2010; Muzzin et al. 2013b). As a consequence, $8 \mathrm{Gyr}$ ago galaxies must have had very different stellar populations. Yet, we have not diagnosed spectroscopically those properties for samples of more than a few dozen individual objects (Jørgensen et al. 2005; Jørgensen \& Chiboucas 2013; Choi et al. 2014; Gallazzi et al. 2014; Belli et al. 2015), and we do not know what processes drive the star formation and assembly history of the galaxy population. The LEGA-C spectra provide us with a unique opportunity to combine the advantages of the "archaeological" approach to galaxy formation with the power of the lookback approach.

We know that galaxies at $z \sim 1$ (and beyond) show a wide variety in structure and star formation activity (e.g., Conselice et al. 2011; Mortlock et al. 2013), but also that, like in the present-day universe, these properties are correlated (e.g., 
Trujillo et al. 2006; Franx et al. 2008; Bell et al. 2012; van der Wel et al. 2014). Knowledge of the stellar ages and metallicities is crucial to understand the star formation history, and stellar velocity dispersions are a crucial element in quantifying the dynamical scaling relations such as the Faber \& Jackson (1976) relation, the Tully \& Fisher (1977) relation, and the fundamental plane (Djorgovski \& Davis 1987; Dressler et al. 1987), the evolution of which has proved to be key in constraining galaxy formation models (e.g., Franx 1993; van Dokkum et al. 1998; Holden et al. 2005; Treu et al. 2005; van der Wel et al. 2005; Bezanson et al. 2013, 2015). Furthermore, the underlying relationships between the stellar content of galaxies and their mass, ongoing star formation, internal structure, environment, and nuclear activity can be explored at large lookback time for the first time by the LEGA-C data set.

The LEGA-C survey can play a crucial and unique role in the context of previous, current, and upcoming developments in the field of galaxy evolution. Wide-area surveys with the Hubble Space Telescope (HST) such as COSMOS (Scoville et al. 2007) and CANDELS (Grogin et al. 2011; Koekemoer et al. 2011) have been completed, producing a high-resolution imaging data set, essentially "frozen" until the James Webb Space Telescope (JWST) and Euclid start operation. After several years with a focus on deep imaging campaigns, the deep spectroscopic effort to probe the stellar light of distant galaxies has fallen behind. Furthermore, ALMA is measuring cold gas masses for increasingly large samples of galaxies (e.g., Carilli \& Walter 2013; Decarli et al. 2014; Scoville et al. 2015). The interplay between stellar populations, ongoing star formation and the available reservoirs for future star formation make for a powerful combination to reconstruct and predict galaxy evolution. Finally, in 2019 the JWST should start operation, marking the beginning of a new era in which JWST and new, large ground-based telescopes will explore the physical properties of galaxies at $z \sim 2$ and beyond. Because the vast majority of all stars formed between $z \sim 2$ and the present, connecting the galaxy populations at $z>2$ and the present is an intractable problem without the intermediate redshift benchmark sample that LEGA-C provides. Yet, JWST is lacking spectroscopic capabilities at $\lambda<1 \mu \mathrm{m}$, leaving many established spectral diagnostics of stellar populations inaccessible; crucially LEGA-C fills this gap.

The main goal of this paper is to introduce the LEGA-C survey. In particular, we outline its scientific goals in Section 2, describe in detail the survey design, observational strategy, and data reduction/analysis in Section 3, and present first-look results in Section 4.

\section{GOALS OF LEGA-C}

The LEGA-C observations produce $\mathrm{S} / \mathrm{N}>10 \AA^{-1}$ spectra for $\sim 3200 \mathrm{~K}$-band selected galaxies with redshifts in the range $0.6<z<1.0$ (or a lookback time of 6-8 Gyr) and over the wavelength range $\sim 6300 \AA-8800 \AA$. Our simple $K$-band selection (see below) is close to a stellar mass selection and is blind to almost any other galaxy property. We chose a $K$ band limit instead of a (model-dependent) stellar mass limit to conserve the legacy value of the data set even if our current methods to estimate stellar masses were to prove incomplete or systematically incorrect. The LEGA-C spectra provide accurate measurements of the following:

\section{Balmer absorption and emission line strengths;}

2. Metal absorption and emission features;

3. Stellar and ionized gas velocity dispersions;

4. Emission line fluxes, widths, and ratios.

These measurements have a wide range of applications that are of interest to a large segment of the community, and each of the topics described below constitutes a large science program. Here, we only list the main features of each science case.

\subsection{Stellar Population Ages}

The strength of the Balmer absorption lines and the spectral region around D4000 are the most sensitive diagnostics of stellar population age (e.g., Kauffmann et al. 2003b; Wild et al. 2009). The age distribution of stellar populations at a lookback time of 6-8 Gyr is one of the main legacy data products of the survey. Gallazzi et al. (2005) and Thomas et al. (2010) measured the age distribution for present-day galaxies. Combined with the observed distribution of star formation across the mass function (e.g., Karim et al. 2011) this can be used to predict the evolution of the age distribution under different assumptions (growth through in situ star formation only; merging). By measuring the distribution of stellar ages 7 Gyr ago (e.g., Gallazzi et al. 2014) the creation of stellar mass and its migration across the mass function can be reconstructed. In particular, this allows us to address the following, interrelated topics.

\subsubsection{Quenching}

The continuous increase in the number density of passive galaxies from $z=1$ to the present (e.g., Bell et al. 2004; Faber et al. 2007) implies that significant numbers of star-forming galaxies have their star-forming activity quenched and then largely remain quiescent. What causes this and how quickly this happens, and whether it is preceded by a burst of star formation is widely discussed, but remains an unsettled problem.

AGN feedback, owing to large available energies and evidence for AGN driven outflows and bubbles (e.g., McNamara \& Nulsen 2007; Nesvadba et al. 2008; Genzel et al. 2014), has long been a strong candidate for driving Galaxy wide quenching of star formation (e.g., Di Matteo et al. 2005; Croton et al. 2006; Hopkins et al. 2006). This idea is consistent with the correlation of quiescence with the amount of stellar mass in galaxy centers: concentration (Kauffmann et al. 2003c; Bell 2008; Franx et al. 2008; Bell et al. 2012), velocity dispersion (Franx et al. 2008; Wake et al. 2012), central stellar mass surface density (Fang et al. 2013), or bulge mass (Lang et al. 2014); in the local universe all these quantities correlate strongly with black hole mass, lending support to the AGN feedback picture. However, a direct causal connection has been difficult to prove, and perhaps the mere presence of a dense body of evolved stars prevents the formation of a large number of new stars (e.g., Martig et al. 2009; Conroy et al. 2015).

The demographics of recently quenched galaxies shed light on this: the fraction of galaxies displaying spectral signs of rapidly reduced recent star formation activity and preceding bursts (e.g., strong Balmer absorption lines, but no emission lines_e.g., Wild et al. (2009)—constrains the quenching rate, speed, and mode. 


\subsubsection{Star Formation and Galaxy Structure}

The connection between quiescence and galaxy structure is a specific instance of a general connection between star formation history and galaxy structure: among star-forming galaxies the instantaneous SFR depends on concentration (Whitaker et al. 2015), and it has been argued that bulge fraction is the driving factor (Abramson et al. 2014; Lang et al. 2014). This explains to some extent, but not fully, the scatter in SFR at fixed galaxy mass. With age measurements for star-forming galaxies the contribution of temporal variations in SFR to the scatter in the so-called star-forming Main Sequence (Noeske et al. 2007) can be constrained. Furthermore, as ALMA will start measuring cold gas masses of large samples of galaxies (Decarli et al. 2014; Scoville et al. 2015) the correlations between star formation, galactic structure and gas fraction provide additional constraints on the short- and longterm variations in star formation activity.

\subsection{Metallicities}

The LEGA-C data set provides the first simultaneous systematic measurement of the stellar and gas phase metal content of the galaxy population at a large lookback time.

\subsubsection{Stellar Metallicity}

The strength of stellar absorption features such as the Fe and $\mathrm{Mg}$ lines constrains the metal content and hence the metal enrichment history of galaxies as they are produced at different stages of the evolution of a stellar population. Through comparison with present-day galaxy population, and in combination with age measurements, the abundance measurements put new and independent constraints on the amount of star formation and chemical enrichment of galaxies over the past 8 Gyr. Previous studies at $z>0.5$ were limited to small samples of very high-mass galaxies or used co-added spectra (Schiavon et al. 2006; Jørgensen \& Chiboucas 2013; Gallazzi et al. 2014). With the large dynamic range in mass and larger sample size we can now measure the intrinsic scatter and mass dependence, which are crucial for interpreting the redshift evolution of the mass-metallicity relation. For several hundred spectra with $\mathrm{S} / \mathrm{N}>30 \AA^{-1}$ the $\alpha / \mathrm{Fe}$ abundance ratio can be quantified. This reveals to what extent the most massive galaxies have had their stellar populations diluted by late star formation and the accretion of externally formed stars (mergers).

\subsubsection{Gas Phase Metallicity}

The standard indicators of the oxygen abundance (as a proxy of gas-phase metallicity) $\mathrm{H} \beta,\left[\mathrm{O}_{\mathrm{II}}\right] \lambda 3727$, and [O $\left.\mathrm{III}\right] \lambda 5007$ (Kewley \& Ellison 2008) are available for the $\sim 1300$ galaxies at redshift $z \sim 0.8$ and below. For the remainder one must rely on the other Balmer lines and $\left[\mathrm{O}_{\mathrm{II}}\right] \lambda 3727$. The detection of (or useful upper limit on) the faint $\left[\mathrm{O}_{\mathrm{III}}\right] \lambda 4363$ line in the deep spectra constrains the electron temperature and therefore breaks the degeneracy between metallicity and electron temperature.

\subsection{Kinematics}

\subsubsection{Dynamical Masses}

Our knowledge of the evolution of the mass function of galaxies relies on photometry-based stellar mass estimates, which depend on a range of assumptions regarding the star formation history, the metal content, the dust content (and its extinction law and geometry), and the stellar initial mass function. Comparisons with dynamical mass measurements have been carried out across galaxies of different types in the present-day universe (e.g., Gallazzi et al. 2006; Taylor et al. 2010), but the necessary measurements (stellar velocity dispersions) have so far been lacking at higher redshifts, with the exception of small samples of massive early-type galaxies (Rettura et al. 2006; van der Wel et al. 2006; Kannappan \& Gawiser 2007; van de Sande et al. 2015).

The 5\%-10\% accurate stellar velocity dispersions from the high-signal-to-noise ratio $(\mathrm{S} / \mathrm{N})$, high-resolution $\left(\sim 50 \mathrm{~km} \mathrm{~s}^{-1}\right)$ LEGA-C spectra, combined with HST-based sizes and Sérsic indices, provide precise dynamical mass measurements. The dynamical masses calibrate stellar mass measurements. Furthermore, differences between dynamical and stellar mass estimates can constrain contributions from dark matter, gas, and IMF variations, and be distinguished by means of cold gas measurements from ALMA, increasing further the accuracy of the work based on gas dynamics (e.g., Erb et al. 2006; Epinat et al. 2009; Förster Schreiber et al. 2009; Law et al. 2009; Erb et al. 2014).

\subsubsection{Scaling Relations}

The measurement of stellar velocity dispersions and the already available structural parameters from HST imaging allow a detailed description of the dynamical scaling relations. The Faber \& Jackson (1976) relation and fundamental plane (Djorgovski \& Davis 1987; Dressler et al. 1987) for early-type galaxies are mapped out at $z \sim 1$ over a large range in galaxy mass of over an order in magnitude, addressing the lingering dispute on the evolution of the Fundamental Plane tilt (Treu et al. 2005; van der Wel et al. 2005; Holden et al. 2010).

For late-type galaxies the Tully \& Fisher (1977) relation can be reconstructed by statistically deprojecting the emission and/ or absorption line widths to derive the circular velocity (Rix et al. 1997; Kassin et al. 2007; Maseda et al. 2013). The LEGA-C data set provides the important opportunity to test ionized gas kinematics with stellar kinematics and calibrate dynamical masses based on emission line widths, building further on previous work (e.g., Vogt et al. 1996; Ziegler et al. 2002; Böhm \& Ziegler 2007; Kassin et al. 2007; Puech et al. 2008; van Starkenburg et al. 2008; Gnerucci et al. 2011; Miller et al. 2011, 2012). The evolution of these scaling relations across $8 \mathrm{Gyr}$ provides stringent constraints on the growth modes of galaxies such as inside-out disk growth (Dutton et al. 2011; Miller et al. 2011) and merging (Naab et al. 2009; Johansson et al. 2012).

\section{DATA}

\subsection{Sample Selection}

The parent sample is the photometric sample from Muzzin et al. (2013a). Their UltraVISTA $K$-band selected catalog is $90 \%$ complete down to $K=23.4$ and contains 160,070 sources across 1.62 square degrees in the COSMOS field (R.A. $=10^{\mathrm{h}}$ $00^{\mathrm{m}}$; decl. $=+2^{\circ} 12^{\prime}$ ). On top of the near-IR VISTA imaging data (McCracken et al. 2012), Muzzin et al. collected and consolidated the available photometric information across 30 passbands ranging over 2 orders of magnitude in wavelength, from 0.2 to $24 \mu \mathrm{m}$. Photometric redshifts were measured by 
Table 1

Sample Selection

\begin{tabular}{|c|c|c|c|c|}
\hline Sample & Redshift & $K$-band Limit & \# in UltraVISTA & $\#$ in LEGA-C \\
\hline Parent & $\ldots$ & 23.4 (90\% compl.) & 160,070 & 4,027 \\
\hline Primary & $0.6<z<1.0$ & $<20.7-7.5 \log ((1+z) / 1.8)$ & 9920 & 3142 \\
\hline Filler I & $1.0<z<1.5$ & $<20.4$ & 2224 & 159 \\
\hline Filler II & $0.6<z<1.0$ & $>20.7-7.5 \log ((1+z) / 1.8)$ & 36,499 & 630 \\
\hline Filler III & $1.0<z<1.5$ & $>20.4$ & 37,136 & 96 \\
\hline
\end{tabular}
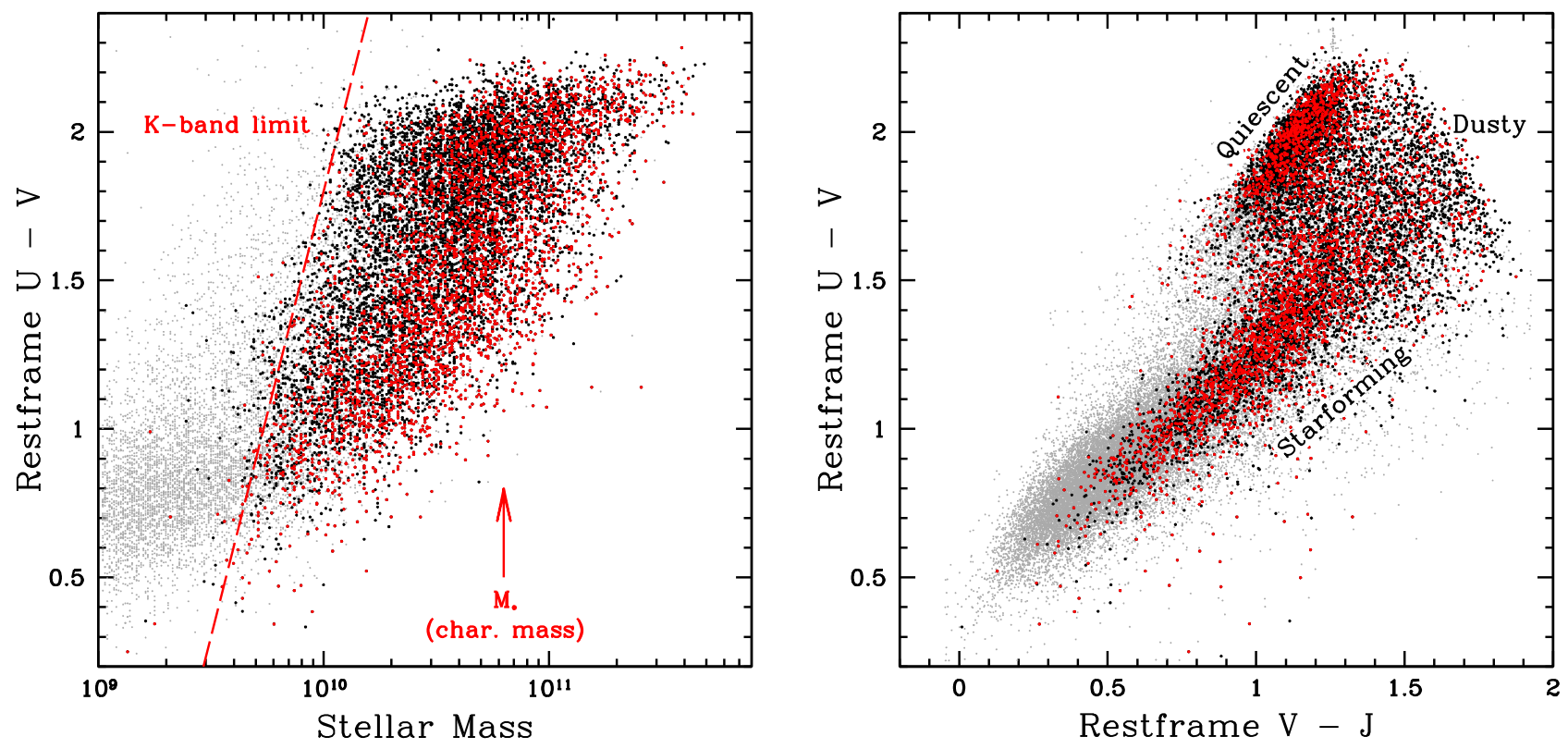

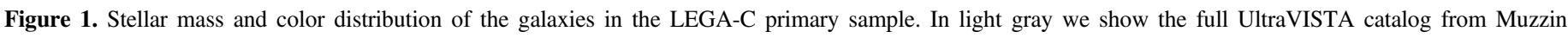

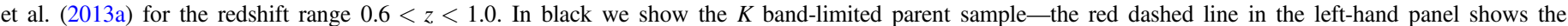

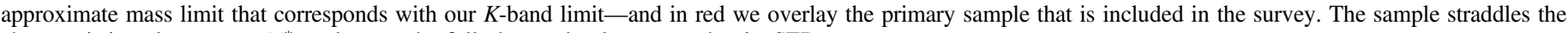
characteristic galaxy mass, $M^{*}$, and spans the full observed color range, that is, SED type.

Muzzin et al. using EAZY (Brammer et al. 2008). Crossmatching with the Davies et al. (2015) catalog results in 21,267 galaxies with spectroscopic redshifts.

Our sample selection procedure is summarized in Table 1. Our primary sample ${ }^{17}$ for the selection of LEGA-C targets consists of 9920 galaxies brighter than $K=20.7-7.5 \times \log ((1+z) / 1.8)$ and $0.6<z<1.0$ (spectroscopic or photometric). That is, our primary sample is $K$ band selected, with a redshift-dependent magnitude limit that ranges from $K=21.08$ at $z=0.6$ to $K=20.7$ at $z=0.8$ to $K=20.36$ at $z=1.0$. The redshift-dependent $K$-band limit ensures that primary targets are sufficiently bright in the spectroscopically observed wavelength range $(0.6 \mu \mathrm{m}-0.9 \mu \mathrm{m})$ to produce a high-quality spectrum, while retaining the advantages of selecting galaxies in the rest-frame near-IR, namely the reduced dependence on variations in age, star formation activity, and extinction.

The basic physical parameters of the galaxies in the primary sample are shown in Figure 1. For all stellar masses larger than $\sim 10^{10} M_{\odot}$ we sample the full range of SED types, from blue to dusty, from star-forming to passive. We note that thanks to our $K$-band selection (rather than, e.g., stellar mass) our sample is

\footnotetext{
17 The primary and filler samples are pre-selected to include only those objects in the UltraVISTA catalog that are not classified as stars and have USE flag with value 1, indicating "good photometric quality" — see Muzzin et al. (2013a) for details.
}

not strongly biased against particular sub-types of galaxies, such as extremely obscured or emission line-dominated objects.

The sample size of $\sim 3200$ and the mass range are motivated by the measurement precision and by the goal of studying the intrinsic scatter in galaxy properties. We require these to be the same: in order to decide whether two sets of galaxies differ in terms of, e.g., age by more than the intrinsic scatter in age, each set needs to contain $\sim 80$ galaxies (for a $3 \sigma$ result). The galaxies span a range in stellar mass of over 1.5 orders of magnitude (Figure 1, left). Given measurement uncertainties of $0.1-0.2$ dex in stellar and dynamical masses, sets of galaxies should be chosen within $\sim 0.3$ dex mass ranges. That is, the survey will probe six independent mass bins. The galaxy population also shows a complex structure in color-color space (Figure 1, right). Given $\sim 0.10-0.15$ mag uncertainties in the colors, sets of galaxies should be chosen within $\sim 0.3$ mag-wide color ranges. Details of the exact definition of color-color bins aside, approximately six independent color-color bins are needed to sample the observed range of SED types. The 6 mass and color-color bins make a total of 36 bins, which each should contain $\sim 80$ galaxies. This motivates our total sample size of about 3000 galaxies.

The required depth of the data, obtained with $20 \mathrm{hr}$ of integration time, is motivated by the required precision of the measurements, which in turn is dictated by the intrinsic 

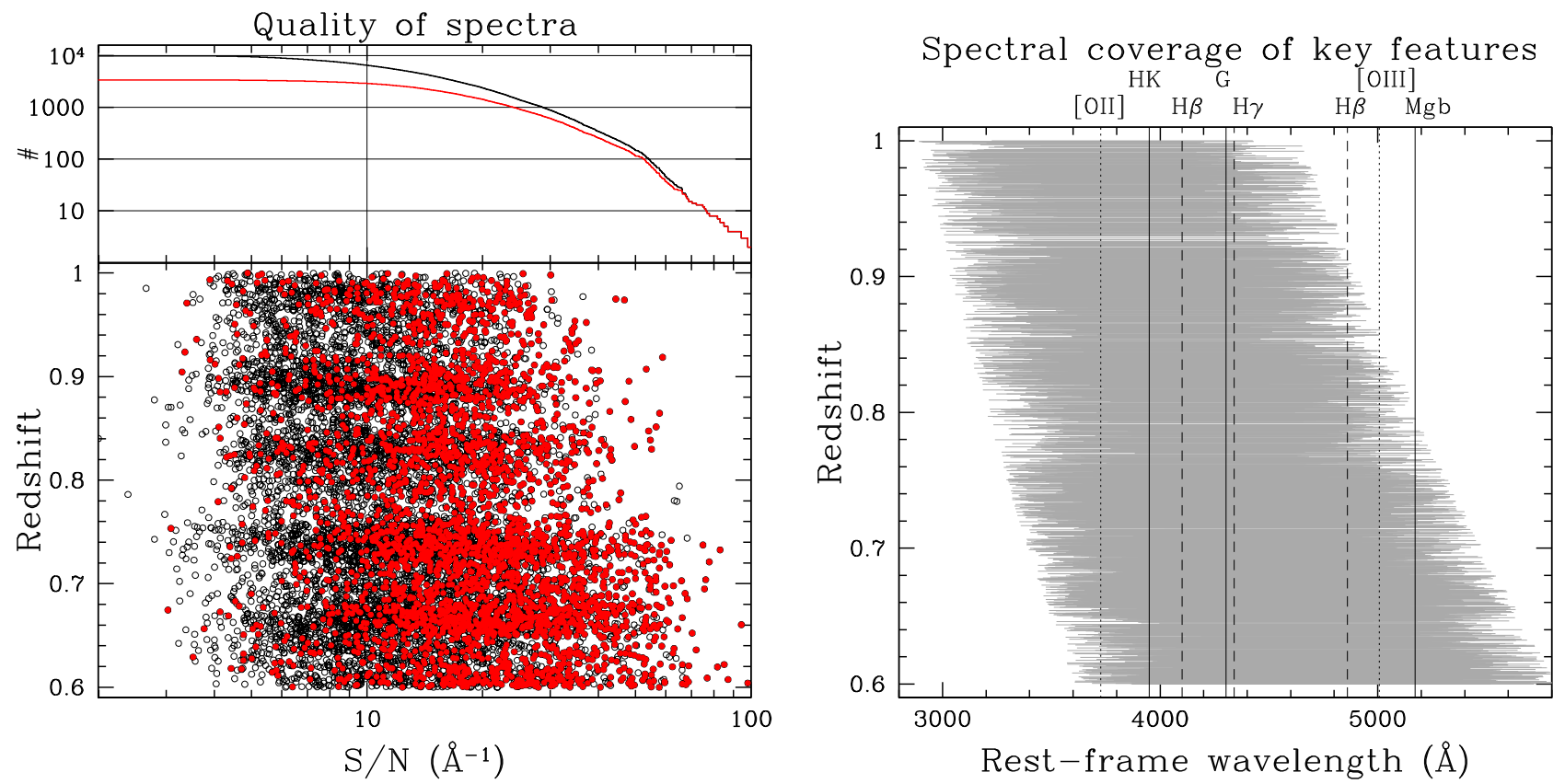

Figure 2. Left: distribution of expected $\mathrm{S} / \mathrm{N}$ vs. redshift for our primary sample. The points represent the $K$ band- selected parent UltraVISTA sample; the red points are those that are in the LEGA-C sample. The top, inset panel shows the cumulative number of galaxies as a function of minimum S/N: black for the parent sample, red for the observed sample. Right: rest-frame wavelength range for the spectra of the observed galaxies. The variation at fixed redshift is due to the variation in slit position. Vertical lines indicate the location of important spectral features.

variation of the physical parameters. For age and metallicity the intrinsic scatter for present-day galaxies is 0.2 dex or more (depending on mass, Gallazzi et al. 2005). That is, we need measurement uncertainties of $0.2 \mathrm{dex}$ or less in these parameters. Similarly, the intrinsic scatter in velocity dispersion for galaxies of a given mass is 0.1 dex (e.g., van der Wel et al. 2008), demanding measurement errors less than that. Our data in hand confirm that the obtained $\mathrm{S} / \mathrm{N}$ is as high as anticipated (Section 4) and sufficient for velocity dispersion, age, and metallicity measurements with a precision of $0.08,0.1$, and 0.2 dex, respectively.

Additional samples are used to fill remaining mask real estate. The filler sample I consists of galaxies at $1.0<z<1.5$ brighter than $K=20.4$; the filler sample II consists of galaxies at $0.6<z<1.0$ but fainter than the (redshift-dependent) $\mathrm{K}$ band limit applied to the primary sample. Any remaining mask space is used for galaxies in the redshift range $1.0<z<1.5$ and fainter than $K=20.4$ (filler sample III).

\subsection{Mask Design}

Four masks designs are created for each of the $7 ! 0 \times 8 ! 4$ VIMOS detectors. Slits are always in the $\mathrm{N}-\mathrm{S}$ direction, $1^{\prime \prime}$ in width and at least $8^{\prime \prime}$ long. Slit assigment is fully automated and proceeds as follows. First, primary targets more than $100^{\prime \prime}$ away from the detector edges in the dispersion direction - to optimize wavelength coverage ${ }^{18}$-are assigned slits, in order of their $K$-band flux (brightest first). This strategy ensures that we maximize the collected number of photons without biasing the sample toward blue, optically bright galaxies. This first step of slit assignment typically fills $60 \%$ of the mask. Before proceeding with assigning additional slits to galaxies, we include at least one blue star with spectral

\footnotetext{
18 The resulting blue wavelength cutoff ranges uniformly from $5800 \AA$ to $6300 \AA$; the red wavelength cutoff ranges uniformly from $8300 \AA$ to $9400 \AA$.
}

coverage at the longest wavelengths to produce a telluric absorption spectrum. Candidate blue stars are identified on the basis of their SDSS colors $(g-r<0.7)$. Next, galaxies in the primary sample closer to the detector edges than $100^{\prime \prime}$ are included in the masks, increasing the occupation of mask real estate to $\sim 75 \%$. Next, alignment stars are included in all four masks, followed by galaxies from the filler samples (see Table 1). An example of a mask design is shown in Figure 3. These mask designs are fed into ESO's vvmps software package to produce the files required for cutting the masks.

The $\sim 2^{\prime}$ gaps between the detectors and the $100^{\prime \prime}$ regions near the edges of the detector in the wavelength $(\mathrm{E}-\mathrm{W})$ direction that are preferably avoided when assigning slits leave about half of the sky uncovered in a single pointing. However, by interlacing the pointings in the E-W direction, we achieve $100 \%$ coverage in that direction, leaving only the $\sim 2^{\prime}$ detector gaps in the $\mathrm{N}-\mathrm{S}$ direction. The survey consists of 32 pointings (four masks each), covering the 1.6 square degree UltraVISTA footprint, and including a total of 4027 galaxies, 3142 of which are from the primary sample (see Table 1 for details).

\subsection{Observations}

Observations are carried out in visitor mode and in dark time. Table 2 shows past and currently scheduled observing runs in Periods 94-96. Similar runs will be scheduled for Periods 97-101, after which the survey will have been completed, barring large delays due to excessive weather losses or technical problems.

The high-resolution red grating $\left(\mathrm{HR}_{\mathrm{red}}\right)$ is used in combination with the GG475 order separation filter. This provides a typical wavelength range of $\sim 6300 \AA-8800 \AA$ at a resolution of $R=2500$ and a dispersion of $0.6 \AA \mathrm{pix}^{-1}$.

For each pointing, we obtain 15-20 observation blocks (OBs) of $4 \times 900-1200 \mathrm{~s}$ exposures each; the total integration time per mask is $20 \mathrm{hr}$. Occasionally an OB will be repeated as 

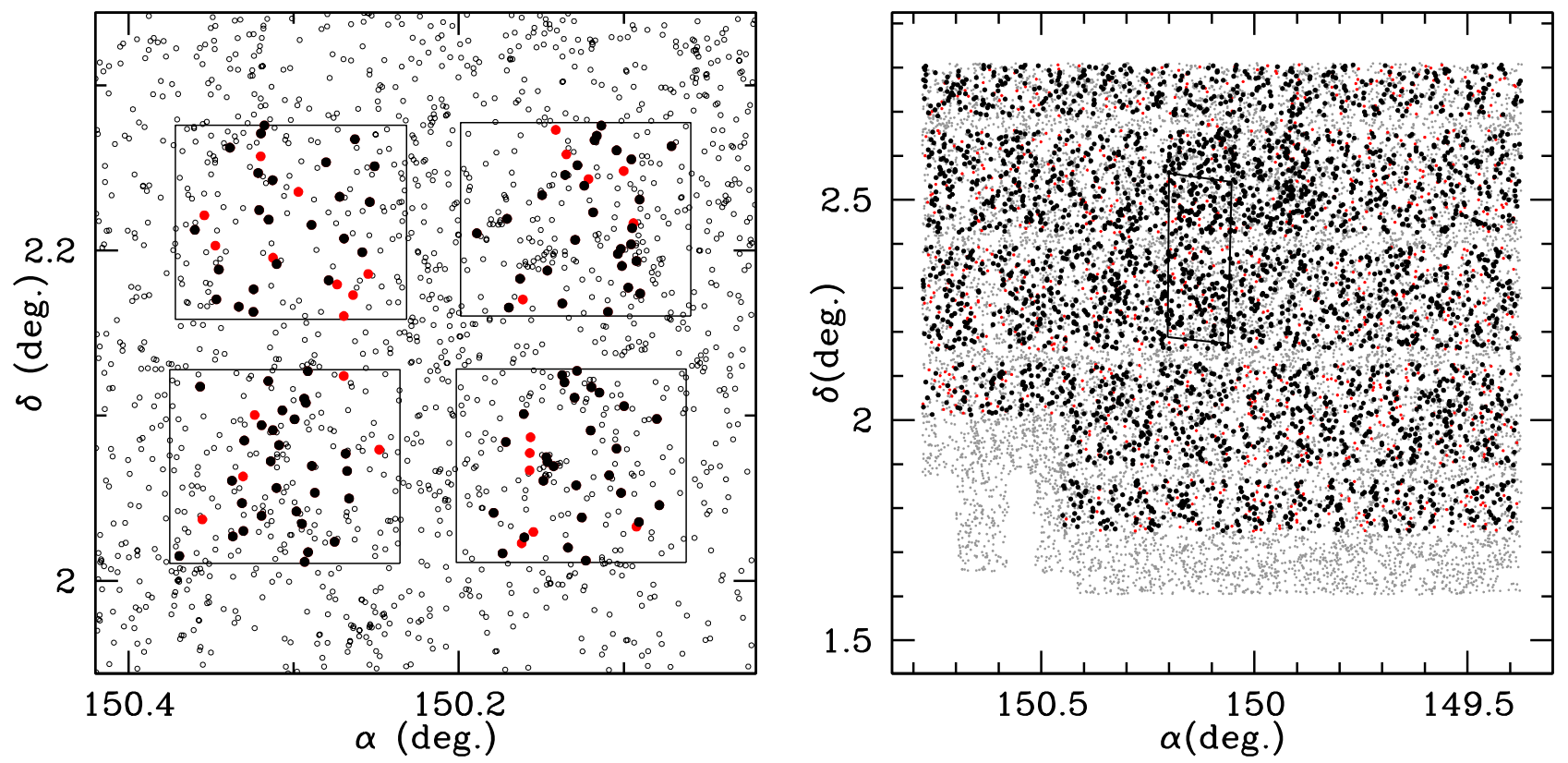

Figure 3. Left: example of mask design for 1 out of 32 pointings. The four squares delineate the footprint of the four VIMOS detectors. The dispersion direction is horizontal (the R.A. direction). The small open circles indicate the full primary sample; the larger, filled circles indicate those that have been given slits in this mask. The red symbols are the filler galaxies. Right: full survey design of 32 pointings, with symbols as in the left-hand panel. Incomplete coverage of UltraVISTA leaves the bottom-left corner out of the LEGA-C survey. Instead we use those two pointing to re-sample the CANDELS field (the rectangular outline near the center) and the well-known $z=0.73$ overdensity just to the N-W of the CANDELS field.

Table 2

Summary of Observations until March 2016

\begin{tabular}{|c|c|c|c|}
\hline Run ID & Hours (Sched.) & Hours (Exp.) & Dates \\
\hline \multicolumn{4}{|c|}{ Period 94} \\
\hline 194.A-2005(A) & 20 & 13 & 2014 Dec $22-27$ \\
\hline 194.A-2005(B) & 66 & 39 & 2015 Jan $15-26$ \\
\hline 194.A-2005(C) & 76 & 41 & 2015 Feb 14-24 \\
\hline 194.A-2005(D) & 66 & 17 & 2015 Mar $15-26$ \\
\hline Sum & 228 & 110 & \\
\hline \multicolumn{4}{|c|}{ Period 95} \\
\hline 194.A-2005(E) & 50 & 31 & 2015 Apr 13-23 \\
\hline 194.A-2005(F) & 30 & 20 & 2015 May $12-22$ \\
\hline Sum & 80 & 51 & \\
\hline \multicolumn{4}{|c|}{ Period 96} \\
\hline 194.A-2005(H) & 26 & 17 & 2015 Dec $04-17$ \\
\hline 194.A-2005(J) & 50 & 32 & 2016 Jan 03-15 \\
\hline 194.A-2005(G) & 36 & 20 & 2016 Feb 02-14 \\
\hline 194.A-2005(I) & 72 & 51 & 2016 Mar 02-14 \\
\hline Sum & 184 & 120 & \\
\hline
\end{tabular}

Note. Scheduled hours correspond to allocated time; integrated hours refer to the actual on-target exposure time.

a result of degrading weather conditions and integration times shortened for scheduling efficiency. Given the large angular sizes of many of our targets a dithering scheme would require slits that are $12^{\prime \prime}-20^{\prime \prime}$ long (proportional to the number of offsets), compared to the minimum of $8^{\prime \prime}$ that we adopt in our survey. In addition, by using the noisy sky as a background measure we would sacrifice $16 \%-30 \%$ in depth (inversely proportional to the number of offsets), equivalent to losing $6-10 \mathrm{hr}$ of exposure time. Combining these factors, dithering would reduce the survey efficiency by several factors. Fortunately, the low fringing amplitudes of the red-sensitive detectors that were installed in 2008 allow us to observe without dithering. The remaining fringing level and problems with the subtraction of the brightest atmospheric emission lines for a subset of targets are merely a cosmetic problem (see Section 3.4) and are a far superior choice compared to the cost of the arguably cosmetically superior dithering approach.

To minimize overheads we deviate from the standard procedure to obtain calibration data (flats and arcs) immediately after the completion of an $\mathrm{OB}$, as this would require slewing the telescope to zenith. Instead, for the data taken between 2014 December and 2015 May we collected calibration data in batches at a series of rotator angles that correspond with the rotator angles during the observation of the science data. This approach intends to limit residual effects of changes in flexure and hysteresis to a level that can be appropriately dealt with during the data reduction process. Careful analysis of these calibration data sets then revealed that proximity in time elapsed between science and calibration data is more relevant than similarity in rotator angle. Therefore, we changed our strategy and obtain a calibration data set after each block of 2-2.5 hr science exposures.

\subsection{Data Reduction}

The data reduction procedure is split into two parts. First, we use the ESO-provided pipeline to produce rectified, wavelength-calibrated 2D spectra. Second, with a custom-made pipeline we improve the wavelength calibration, perform simultaneous sky subtraction and object extraction, and coadd the individual frames. 


\subsubsection{ESO Pipeline}

The Reflex interface is used to run the ESO-provided pipeline recipes on the data from an individual OB consisting of four exposures, producing a single, averaged frame. The steps performed with the ESO pipeline include a bias and flatfield correction, slit definition, slit rectification, wavelength calibration, spectro-photometric calibration, and cosmic ray rejection.

\subsubsection{Custom Pipeline}

There are three main reasons for switching to a custom-made set of data reduction recipes at this point to further process the OB-level, combined exposures. First, the VIMOS mask design software forces gaps between the slits of only 2 pixels $(0 ! 4)$, causing bleeding of bright skylines into the neighboring slits, making sky subtraction more difficult. Second, the alignment between the science and calibration frames is imperfect due to flexure, which requires reducing the length of the slits across which sky subtraction is performed. Third, our non-dithering strategy outlined above requires that object extraction and sky subtraction are done simultaneously.

2D error spectra are calculated from the flux in the science frames and the read noise level. Slit definitions are automatically verified and adjusted. Using the expected location of a target galaxy as an initial estimate, the size and precise location are measured by fitting a Gaussian profile in the spatial direction after summing along a 1000 pixel range in the wavelength direction. This location is then traced along the entire wavelength range in bins of 100 pixels. For $\sim 10 \%$ of the targets in each mask there is insufficient flux to do this, and the location is assumed to be the expected location and independent of wavelength. Secondary objects in the slits are extracted separately.

The default model for sky+galaxy is a flat background with a Gaussian profile of a fixed width and location (as measured above). That is, the two free parameters are the amplitude of the Gaussian (the galaxy flux) and the sky background level. This model is fit to each individual wavelength bin, across the spatial direction, weighed by the error spectrum.

This simple model does not always produce a sufficiently accurate description of the data, as made apparent by systematic residuals. In such cases a first-order term is added to the background model for areas where the sky level is high or the gradient in the sky level in the wavelength direction is large.

This data reduction process produces individual extracted galaxy spectra and their associated error spectra (for each of the 15-20 OBs, totalling $20 \mathrm{hr}$ of exposure time). Each of these are corrected for telluric absorption features as follows: the continuum of the blue star spectrum is fit by a 5 th order polynomial over the regions that do not contain telluric absorption features, and divided out. All galaxy spectra are then divided by the resulting telluric spectrum. These are then co-added, weighed by the $\mathrm{S} / \mathrm{N}$ as measured in the observed wavelength range $8030 \AA-8270 \AA$, which is relatively devoid of bright sky lines. Finally, the flux is measured over the wavelength range $7000 \AA-8400 \AA$ and normalized to match the photometric $i^{+}$-band flux of the object. This remedies errors in the automatic spectro-photometric calibration that can arise due to varying transparency during the observations. The accuracy of the calibration is better than $5 \%$, but due to varying slit losses (which range from $10 \%-40 \%$ ) the precision varies from galaxy to galaxy. As a higher-level data product we will provide, in the future, a new, wavelength-dependent flux calibration based on matching the shape of the spectrum to that of the full photometric spectroscopic energy distribution, as well as a model for the slit losses.

As mentioned above, our observing strategy is aimed at maximizing the survey efficiency and depth by not dithering, a choice that comes at the cost of retaining some cosmetic flaws as a result of low-level fringing and imperfections in the sky subtraction of the brightest atmospheric emission lines that occur when data are obtained under sub-optimal weather conditions and for objects that are near the edges of the detectors. The resulting features are illustrated in Figure 4. We stress that, in total, only about $2 \%-3 \%$ of the pixels suffer from such problems, and those are wavelength regions where the night sky is bright anyway, implying that only low-S/N data is lost.

\subsection{Spectral Fitting-Redshifts, Stellar Population Properties and Velocity Dispersions}

Redshifts are measured by cross-correlating with a small set of general template spectra. In $98 \%$ of the cases our redshifts are within the targeted range of $z=0.6-1.0$ and are visually verified. Among the $2 \%$ of galaxies that fall outside this range (18 objects), 9 are only marginally lower than $z=0.6$ or higher than $z=1.0(\Delta z \lesssim 0.02)$, and we do not consider their inclusion a mistake. Among the nine others, four had wrong previous spectroscopic redshifts (while the photometric redshifts were indeed correct), presumably due to the poor quality of that previously available spectroscopic data. Four other galaxies, with no pre-existing spectroscopic redshifts, had wrong photometric redshifts: 3 of those were edge-on (dusty) disks. One galaxy-a blend of two galaxies at different redshifts-had a wrong photometric redshift as well as a wrong pre-existing spectroscopic redshift. We conclude that our sample is affected by wrong selection redshifts at the $1 \%$ level.

The key measurements made from the spectra are the stellar population age and metallicity, and the stellar/gas velocity dispersion. Here we provide a brief overview of the methodology, while full descriptions of the testing and error budget are postponed until these measurements are made publicly available (end of 2016).

The first step is to fit the full spectrum following the method of Pacifici et al. (2012). Star formation and chemical enrichment histories from the De Lucia \& Blaizot (2007) galaxy formation model are used to generate a library of template spectra, where the evolving chemical enrichment is calculated based upon the star formation history. We estimate gas-phase metallicities as well and CLOUDY (Ferland et al. 1998) is used to calculate the contribution of nebular line and continuum emission in a manner consistent with the radiation output of the stars. We adopt the model of Charlot \& Fall (2000) to treat dust, where the light from old stars is attenuated by a large-scale,diffuse component, and the light from young stars is attenuated first by a birth-cloud component and then by the diffuse component.

Given the restframe spectrum from which the continuum has been removed, likelihoods are computed for all models, resulting in marginalized probability distributions for the model parameters. The quantities of interest here are specific 


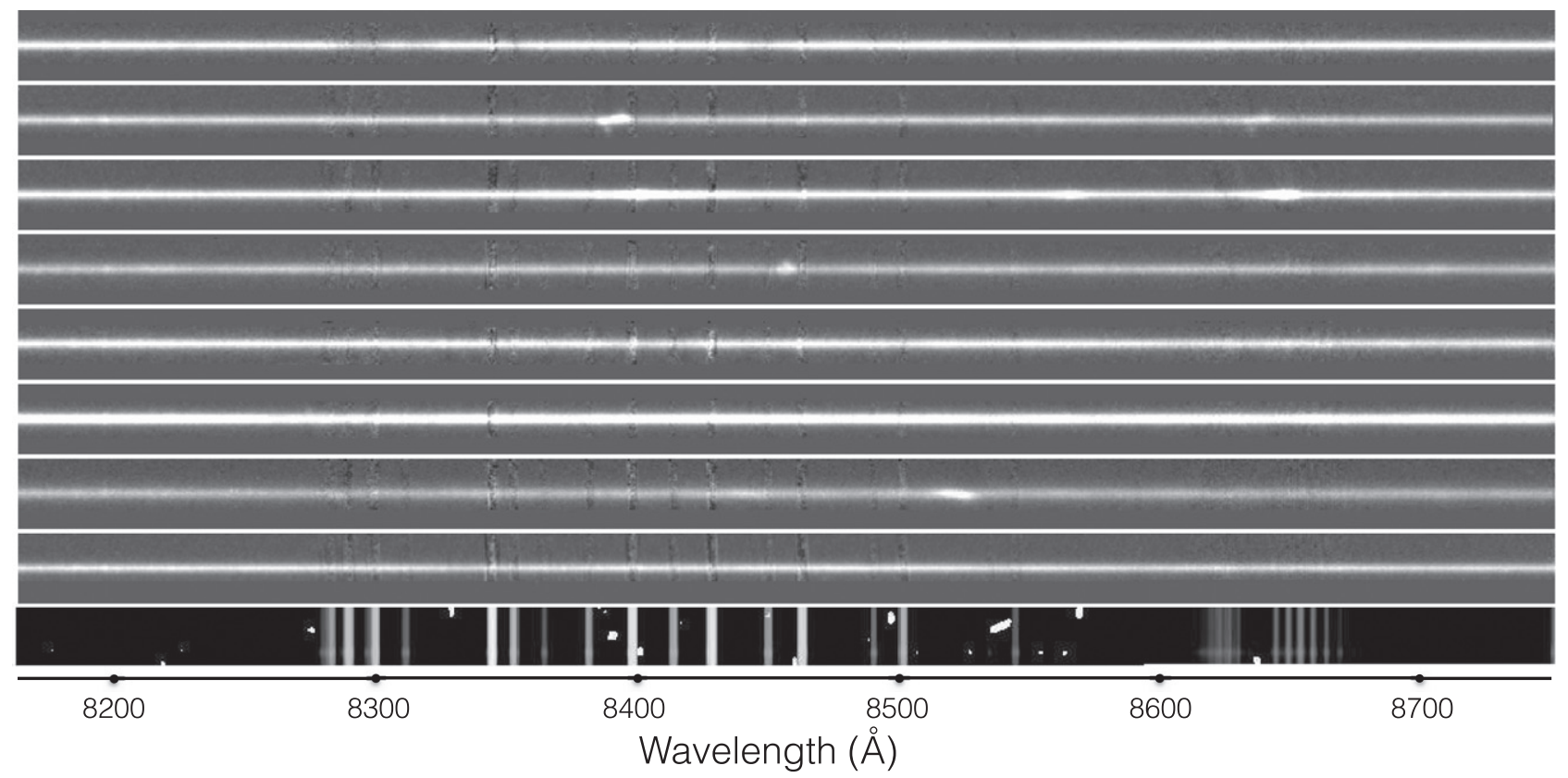

Figure 4. To illustrate the $\mathrm{S} / \mathrm{N}$ of the data and the quality of the sky subtraction we show co-added two-dimensional spectra of eight galaxies over the wavelength range $8260 \AA-8760 \AA$, chosen for its bright, telluric emission lines. The bottom row shows the variance spectrum of the galaxy spectrum right above it, to indicate which parts are clean and which parts are affected by the telluric lines. These eight examples show the full range in the quality of sky subraction. The quality of the sky subtraction (see Section 3.4.2 for a description of the algorithm) at the locations of the brightest atmospheric features varies from galaxy to galaxy due to imperfections in the calibration data (bottom two spectra) or low-level fringing (third from the top). In other cases the sky subtraction is noise limited. We note that even for the worst cases only a few dozen pixels across the entire spectrum are not noise limited.

star formation rate, luminosity-and mass-weighted age, gasphase, and stellar metallicity.

The 10 best-fitting model spectra from this full spectral fit of a given galaxy spectrum are used as templates for the velocity dispersion measurement for that galaxy, following the approach of Bezanson et al. (2015). Using the pPXF code (Cappellari \& Emsellem 2004), emission-line subtracted galaxy and model spectra are continuum filtered by multiplicative and additive polynomials, after which a Gaussian convolution of the model spectra in real space is fit to the galaxy spectrum. The gasphase velocity dispersion is estimated by fitting the continuum subtracted galaxy spectrum with the best-fitting emission line model spectrum, convolved with a Gaussian. We note that these kinematic quantities are integral measurements from the light in the slits-no aperature corrections are made at this point.

The resulting stellar velocity dispersion is then used to smooth the model spectra used for the spectral fitting and the initial stellar population fit is repeated to verify that the initial results were not biased due to the mismatch in spectral resolution.

\subsubsection{Stellar Populations: Line Indices}

Measurement and interpretation of stellar absorption features in the optical range provide a well-tested and alternative method to full spectral fitting to derive constraints on stellar mass, ages and abundances. In particular we measure all the indices defined in the Lick/IDS system (Worthey et al. 1994; Worthey \& Ottaviani 1997), including the high-order Balmer lines, in the galaxy spectra cleaned of emission lines (obtained by subtracting the best-fit emission line model spectrum as described in Section 3.5). Following the approach outlined in Gallazzi et al. $(2005,2014)$, we derive marginalized probability density functions for stellar mass, light- and mass-weighted stellar ages and stellar metallicities by comparing the observed strengths (accounting for velocity dispersion broadening) of optimal sets of absorption indices (defined on the basis of the galaxy redshift) to a library of $\mathrm{BC} 03$-based models convolved with stochastic SFHs and metallicities. Additionally, element abundance ratios are estimated from the ratio of $\mathrm{Mg}$ and $\mathrm{Fe}$ features (Gallazzi et al. 2006, e.g., $\mathrm{Mgb} /\langle\mathrm{Fe}\rangle$ ) and from $\mathrm{CN}$ indices (e.g., Carretero et al. 2004).

\subsection{Auxiliary Data sets}

\subsubsection{Photometry: Stellar Masses and Rest-frame Colors}

Using the data from Muzzin et al. (2013a, 2013b) and following their methodology we use FAST (Kriek et al. 2008) to fit the photometric spectral energy distribution and measure stellar masses and rest-frame colors. Here, a template library with solar-metallicity spectra from SFHs with exponentially declining SFRs and dust-screen attenuation (Calzetti et al. 2000) is used. The improvements with respect to the modeling results from Muzzin et al. (2013b) stem solely from the use of our spectroscopic redshifts. In a second iteration we jointly fit the photometry and spectroscopy, deriving stellar masses and rest-frame colors with the methodology described above in Section 3.5. We will provide both photometric and photometric+spectroscopic stellar mass estimates.

\subsubsection{Structural Parameters from HST Imaging}

ACS imaging is available for nearly all targets from the COSMOS program (Scoville et al. 2007). 10" image cutouts are created with the IPAC tool and the 2-D light profiles are fit with single-Sérsic profiles using galfit (Peng et al. 2010), with effective radius, total magnitude, Sérsic index, axis ratio, 


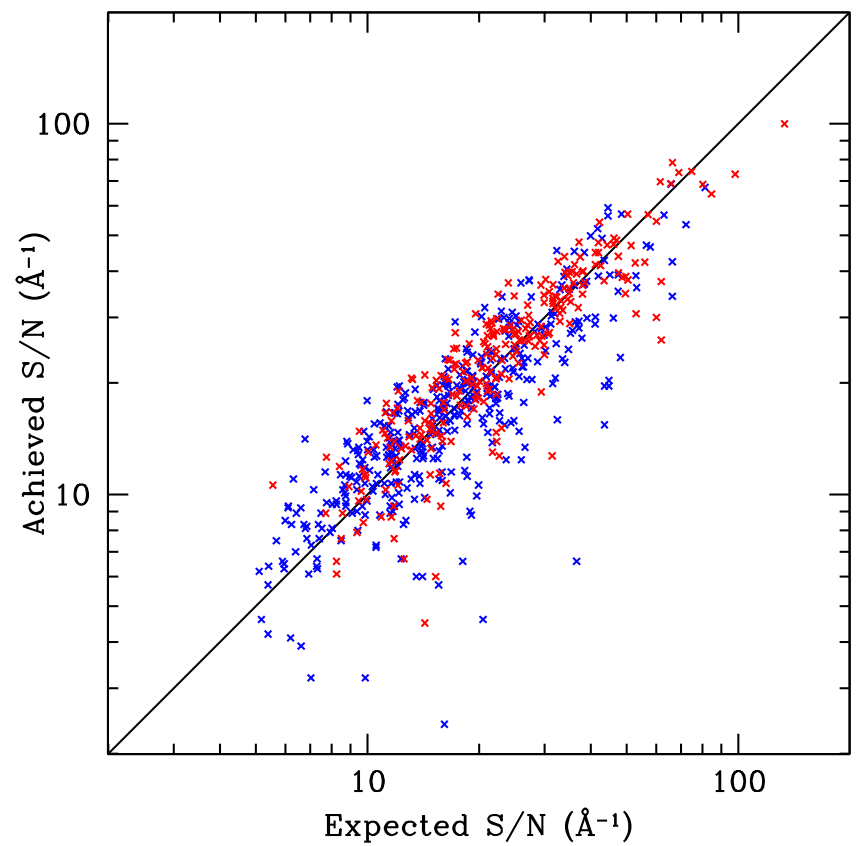

Figure 5. Measured S/N per $\AA$ (averaged over $8030 \AA-8270 \AA$ ) for the first year of data vs. the expected $\mathrm{S} / \mathrm{N}$, based on $i^{+}$band magnitude and galaxy size, calculated in preparation of the survey. Blue crosses indicate star-forming galaxies, red crosses quiescent galaxies. The general agreement implies that our predictions were accurate and that the data quality is as expected, if not slightly better. The scatter can be explained by variations in radial surface brightness profiles, axis ratios, position angles, and observing conditions.

and position angle, and background as the free parameters. The Point Spread Function is chosen from a list of stars in the COSMOS field based on closest proximity in terms of detector position. A user-created error image is calculated based on the IPAC-provided weight image with added Poisson noise from the objects. Neighbors are simultaneously fit. Uncertainties are assigned based on the total $\mathrm{S} / \mathrm{N}$ and values of the fitting parameters, as described by van der Wel et al. (2012).

\section{FIRST LOOK RESULTS}

An early milestone in the LEGA-C survey is to confirm that the data quality is as expected. In Figure 5 we show the $\mathrm{S} / \mathrm{N}$ distribution for the primary sample observed in 2014-2015, in comparison with the expected $\mathrm{S} / \mathrm{N}$ that we calculated in advance. For star-forming galaxies we typically achieve $\mathrm{S} / \mathrm{N}=10-20 \AA^{-1}$ and for quiescent galaxies $\mathrm{S} / \mathrm{N}=20-30 \AA^{1}$, both in line with the expected values.

In Figure 6 we show for the primary sample observed so far ( $23 \%$ of the total) the bi-modal distribution in terms of stellar mass and star formation rate (broad-band photometry-based, from Muzzin et al. 2013b). ${ }^{19}$ The dynamic range in these quantities is such that our sample represents the full galaxy population with stellar masses $M_{*}>10^{10} M_{\odot}$, where the majority of stars form and live throughout cosmic history (e.g., Karim et al. 2011).

An illustration of the application of new information obtained through our deep spectroscopy is shown in Figure 6, where we color-code the objects by their stellar velocity

\footnotetext{
${ }^{19}$ We note that the star formation rate indicator is based on UV and IR fluxes. Therefore, any source of UV+IR radiation that is not from star formation is still counted as such. For example, dust heated by evolved stellar populations may significantly affect the IR luminosity of massive early-type galaxies (e.g., Groves et al. 2012).
}

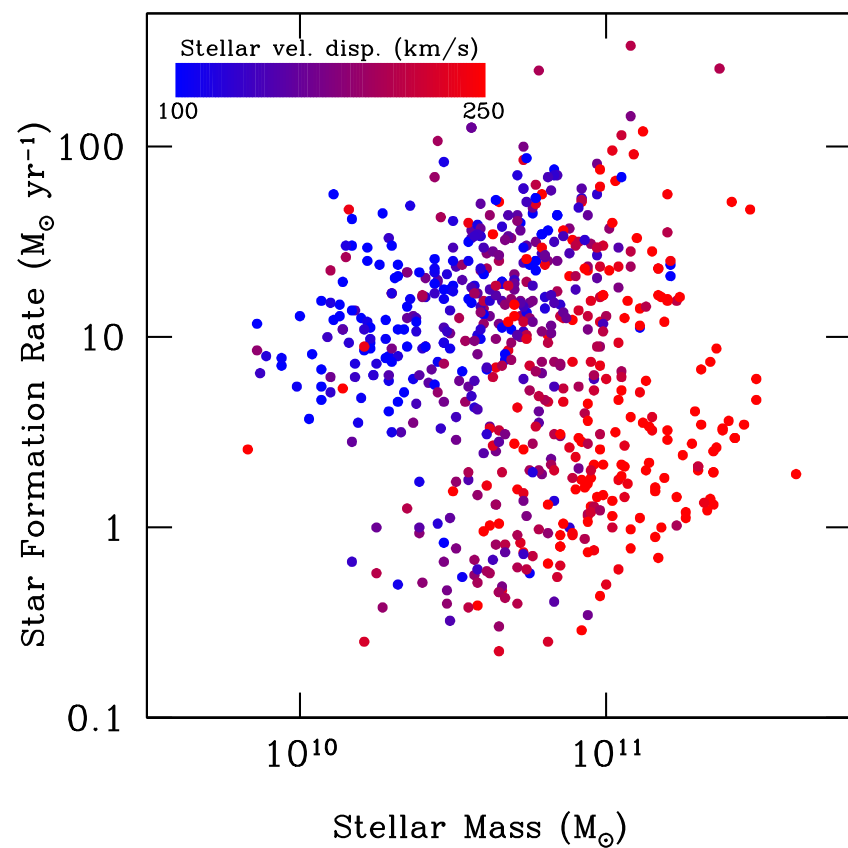

Figure 6. Stellar mass vs. UV+IR-based SFR for the LEGA-C 1st year primary sample (653 galaxies). The color-coding represents stellar velocity dispersion.

dispersion. Galaxies with higher stellar masses have higher velocity dispersions, but also, at a fixed stellar mass, quiescent galaxies have higher velocity dispersions than star-forming galaxies. This has been seen for present-day galaxies (Wake et al. 2012) and has been predicted for high-redshift galaxies (Franx et al. 2008). Our evidence that star formation activity correlates with dynamical structure implies that the different size-mass relations for star-forming and quiescent galaxies (e.g., Shen et al. 2003; van der Wel et al. 2014) provide important constraints on galaxy formation models, do not merely reflect differences in $M / L$ gradients, and do not result from practical problems with measuring sizes of distant galaxies.

We note that the correlation between true velocity dispersion (meaning the dispersion in stellar velocities at a fixed location in a galaxy) and star formation activity must be even stronger than is apparent in Figure 6: much of our velocity dispersion estimates-simple, 2nd-order velocity moments, measured from the integrated light in the slit-presumably reflect rotation, especially for star-forming disks.

A visual impression of the quality of the spectra, and the range in galaxy properties in the same, is shown in Figure 7. Ten spectra are sorted by the galaxies' Sérsic index, a parameter that, unlike stellar mass or color, is measured independently of spectral properties. Stellar population characteristics are visually apparent for all 10 galaxies, regardless of their structural properties or star formation activity. We see a clear change in spectral properties as a function of structure, from passive/old for high-Sérsic index galaxies to young/starforming for low-Sérsic index galaxies. We note that even the youngest objects show clear absorption lines.

A further illustration is given in Figure 8, where we sort five star-forming disk galaxies by their inclination angles. Face-on and edge-on disks each present their own specific observational challenge for deep spectroscopy: low surface brightness and extinction, respectively. Yet, we have achieved high-S/N continuum spectra for each of the galaxies. 

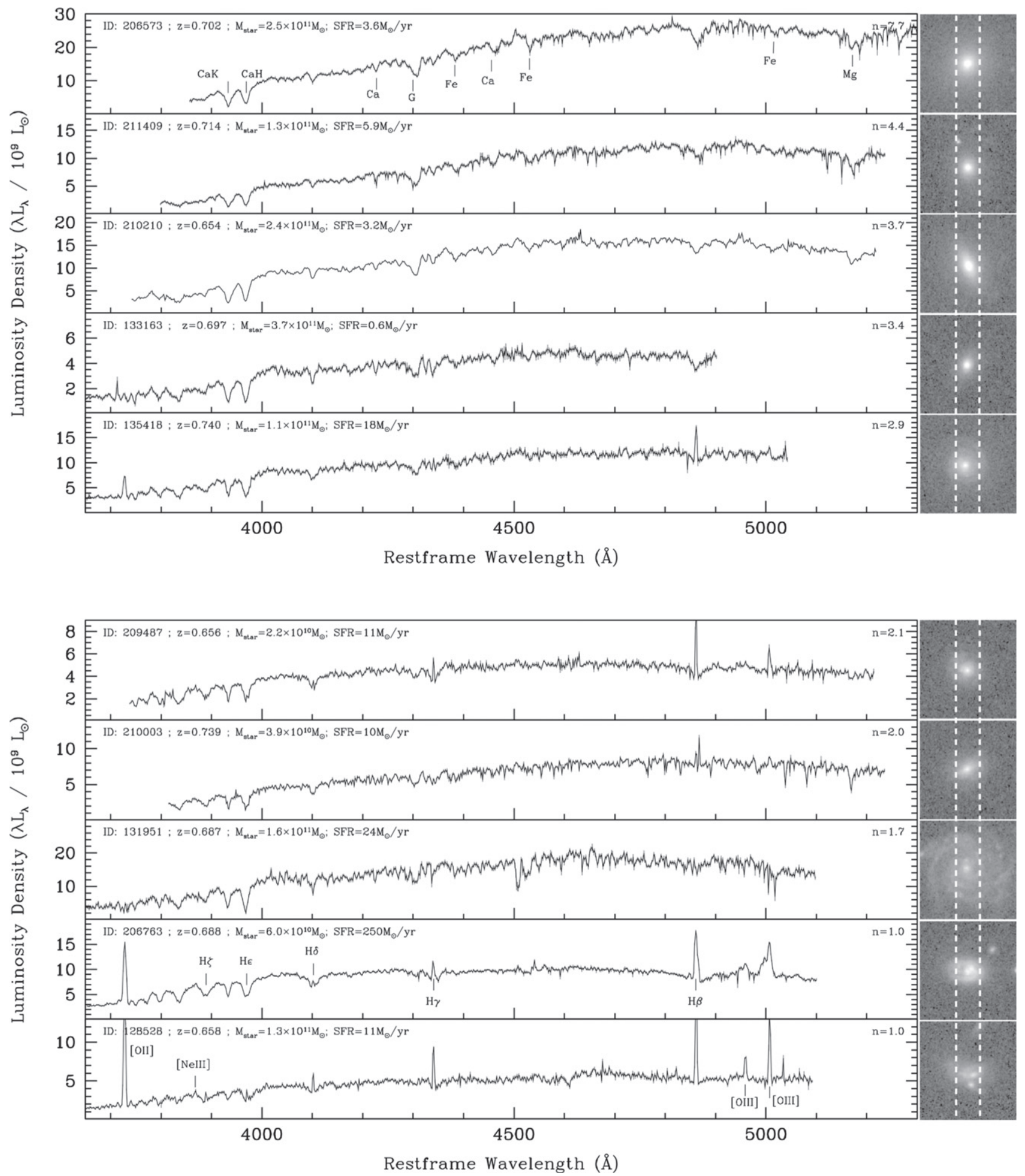

Figure 7. Ten representative LEGA-C spectra sorted by Sérsic index $n$. These spectra have $\mathrm{S} / \mathrm{N}$ close to or better than the survey median; $1 \sigma$ errors are indicated in gray. Metal absorption features are labeled in the top panel; Balmer absorption/emission lines and metal emission lines are labeled in the bottom panels. Identification number, redshift, stellar mass (from broad-band SED fits), and SFR (derived from total UV and mid-IR fluxes) are given in each panel. On the right-hand side HST/ ACS cutouts (4". 5 across) are shown, along with the slit orientation $(\mathrm{N}-\mathrm{S})$ and width $\left(1^{\prime \prime}\right)$. A clear correlation between spectral and structural properties is obvious at first sight: more concentrated galaxies have older populations than less concentrated galaxies. In many cases slit losses are limited and the spectra provide a representative view of the galaxy, but in some cases a significant amount of the light is missed due to the extent and/or orientation of the galaxy (e.g., ID 131951).

\section{TIMELINE AND OUTLOOK}

The LEGA-C observations will nominally conclude in 2018 May, modulo uncertain weather and other losses. The first data release is scheduled for 2016 June 1st. This release will include reduced, calibrated, extracted spectra for all 925 galaxies for which observations were completed before 2015 June. A basic catalog with UltraVISTA IDs, coordinates, magnitudes, redshifts, and $\mathrm{S} / \mathrm{N}$ values will accompany this release. 


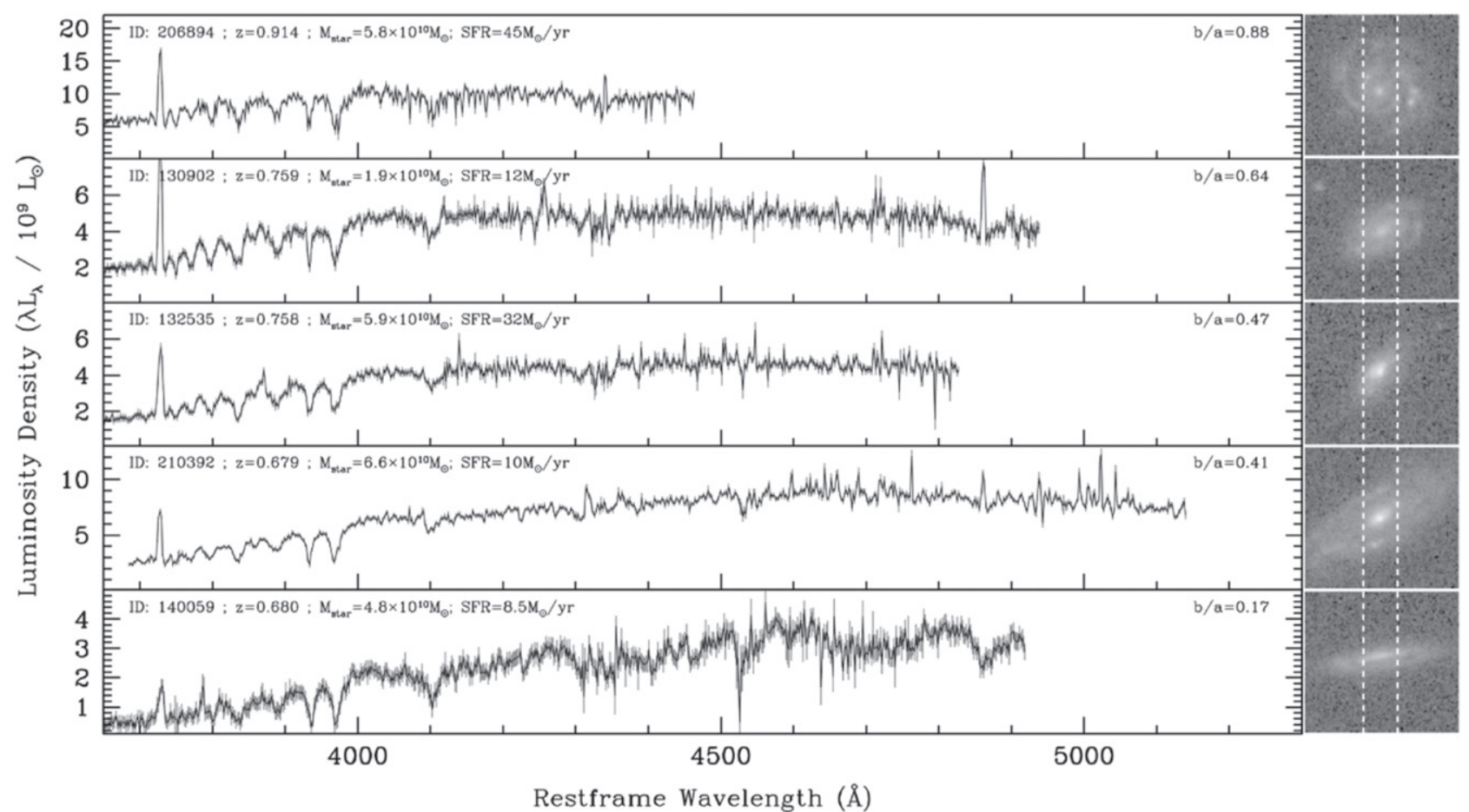

Figure 8. Five LEGA-C spectra of disk galaxies sorted by inclination (projected axis ratio $b / a$ ). See the caption of Figure 7 for a detailed description of the contents of this figure. A clear reddening is seen for more inclined galaxies, and despite significant dust columns the continuum has high S/N in all cases (e.g., ID 140059).

A second release will follow by the end of 2016, which will include spectra for all galaxies observed until 2016 May, as well as the first set of value-added catalogs with redshifts, velocity dispersions, and emission/absorption line strength indices. The third and fourth data releases are scheduled for 2017 December and 2018 December. Besides the additional spectra and the first set of value-added catalogs, these two releases will also include a second set of value-added catalogs containing stellar masses, ages, metallicities, structural parameters from HST imaging and dynamical masses.

With the data in hand, we can, as a community, start addressing scientific questions outlined in Section 2. The data will be publicly released by 2016 June 1 , and we do not have to wait until the sample collection is complete to achieve many science goals. The current data set already far surpasses any previously existing data set.

We thank the referee for a thorough reading of the manuscript and constructive comments. A.W. and K.N. acknowledge support from the Deutsche Forschungsemeinschaft (GZ: WE 4755/4-1). R.B. gratefully acknowledges support by NASA through Hubble Fellowship grants \#HF51318 awarded by the Space Telescope Science Institute, which is operated by the Association of Universities for Research in Astronomy, Inc., for NASA, under contract NAS 5-26555. C.P. acknowledges support by an appointment to the NASA Postdoctoral Program at the Goddard Space Flight Center, administered by USRA through a contract with NASA. We gratfeully acknowledge the NWO Spinoza grant. V.W. acknowledges support from the European Research Council Starting Grant (SEDMorph; P.I. V. Wild).

\section{REFERENCES}

Abramson, L. E., Kelson, D. D., Dressler, A., et al. 2014, ApJL, 785, L36 Bell, E. F. 2008, ApJ, 682, 355
Bell, E. F., van der Wel, A., Papovich, C., et al. 2012, ApJ, 753, 167 Bell, E. F., Wolf, C., Meisenheimer, K., et al. 2004, ApJ, 608, 752 Belli, S., Newman, A. B., \& Ellis, R. S. 2015, ApJ, 799, 206

Bezanson, R., Franx, M., \& van Dokkum, P. G. 2015, ApJ, 799, 148 Bezanson, R., van Dokkum, P. G., van de Sande, J., et al. 2013, ApJL, 779, L21

Böhm, A., \& Ziegler, B. L. 2007, ApJ, 668, 846

Brammer, G. B., van Dokkum, P. G., \& Coppi, P. 2008, ApJ, 686, 1503

Calzetti, D., Armus, L., Bohlin, R. C., et al. 2000, ApJ, 533, 682

Cappellari, M., \& Emsellem, E. 2004, PASP, 116, 138

Carilli, C. L., \& Walter, F. 2013, ARA\&A, 51, 105

Carretero, C., Vazdekis, A., Beckman, J. E., Sánchez-Blázquez, P., \& Gorgas, J. 2004, ApJL, 609, L45

Charlot, S., \& Fall, S. M. 2000, ApJ, 539, 718

Choi, J., Conroy, C., Moustakas, J., et al. 2014, ApJ, 792, 95

Conroy, C., van Dokkum, P. G., \& Kravtsov, A. 2015, ApJ, 803, 77

Conselice, C. J., Bluck, A. F. L., Ravindranath, S., et al. 2011, MNRAS, 417, 2770

Croton, D. J., Springel, V., White, S. D. M., et al. 2006, MNRAS, 365, 11

Davies, L. J. M., Driver, S. P., Robotham, A. S. G., et al. 2015, MNRAS, 447, 1014

De Lucia, G., \& Blaizot, J. 2007, MNRAS, 375, 2

Decarli, R., Walter, F., Carilli, C., et al. 2014, ApJ, 782, 78

Di Matteo, T., Springel, V., \& Hernquist, L. 2005, Natur, 433, 604

Dickinson, M., Papovich, C., Ferguson, H. C., \& Budavári, T. 2003, ApJ, 587,25

Djorgovski, S., \& Davis, M. 1987, ApJ, 313, 59

Dressler, A., Lynden-Bell, D., Burstein, D., et al. 1987, ApJ, 313, 42

Dutton, A. A., van den Bosch, F. C., Faber, S. M., et al. 2011, MNRAS, 410,1660

Epinat, B., Contini, T., Le Fèvre, O., et al. 2009, A\&A, 504, 789

Erb, D. K., Steidel, C. C., Shapley, A. E., et al. 2006, ApJ, 646, 107

Erb, D. K., Steidel, C. C., Trainor, R. F., et al. 2014, ApJ, 795, 33

Faber, S. M., \& Jackson, R. E. 1976, ApJ, 204, 668

Faber, S. M., Willmer, C. N. A., Wolf, C., et al. 2007, ApJ, 665, 265

Fang, J. J., Faber, S. M., Koo, D. C., \& Dekel, A. 2013, ApJ, 776, 63

Ferland, G. J., Korista, K. T., Verner, D. A., et al. 1998, PASP, 110, 761

Förster Schreiber, N. M., Genzel, R., Bouché, N., et al. 2009, ApJ, 706, 1364 Franx, M. 1993, PASP, 105, 1058

Franx, M., van Dokkum, P. G., Schreiber, N. M. F., et al. 2008, ApJ, 688, 770 Gallazzi, A., Bell, E. F., Zibetti, S., Brinchmann, J., \& Kelson, D. D. 2014 ApJ, 788, 72 
Gallazzi, A., Charlot, S., Brinchmann, J., \& White, S. D. M. 2006, MNRAS, 370,1106

Gallazzi, A., Charlot, S., Brinchmann, J., White, S. D. M., \& Tremonti, C. A. 2005, MNRAS, 362, 41

Genzel, R., Förster Schreiber, N. M., Rosario, D., et al. 2014, ApJ, 796, 7

Gnerucci, A., Marconi, A., Cresci, G., et al. 2011, A\&A, 528, A88

Graves, G. J., Faber, S. M., \& Schiavon, R. P. 2009, ApJ, 698, 1590

Grogin, N. A., Kocevski, D. D., Faber, S. M., et al. 2011, ApJS, 197, 35

Groves, B., Krause, O., Sandstrom, K., et al. 2012, MNRAS, 426, 892

Holden, B. P., van der Wel, A., Franx, M., et al. 2005, ApJL, 620, L83

Holden, B. P., van der Wel, A., Kelson, D. D., Franx, M., \& Illingworth, G. D. 2010, ApJ, 724, 714

Hopkins, P. F., Hernquist, L., Cox, T. J., et al. 2006, ApJS, 163, 1

Ilbert, O., Salvato, M., Le Floc'h, E., et al. 2010, ApJ, 709, 644

Johansson, P. H., Naab, T., \& Ostriker, J. P. 2012, ApJ, 754, 115

Jørgensen, I., Bergmann, M., Davies, R., et al. 2005, AJ, 129, 1249

Jørgensen, I., \& Chiboucas, K. 2013, AJ, 145, 77

Kannappan, S. J., \& Gawiser, E. 2007, ApJL, 657, L5

Karim, A., Schinnerer, E., Martínez-Sansigre, A., et al. 2011, ApJ, 730, 61

Kassin, S. A., Weiner, B. J., Faber, S. M., et al. 2007, ApJL, 660, L35

Kauffmann, G., Heckman, T. M., Tremonti, C., et al. 2003a, MNRAS, 346, 1055

Kauffmann, G., Heckman, T. M., White, S. D. M., et al. 2003b, MNRAS, 341,33

Kauffmann, G., Heckman, T. M., White, S. D. M., et al. 2003c, MNRAS, 341,54

Kauffmann, G., White, S. D. M., Heckman, T. M., et al. 2004, MNRAS, 353,713

Kewley, L. J., \& Ellison, S. L. 2008, ApJ, 681, 1183

Koekemoer, A. M., Faber, S. M., Ferguson, H. C., et al. 2011, ApJS, 197, 36

Kriek, M., van der Wel, A., van Dokkum, P. G., Franx, M., \& Illingworth, G. D. 2008, ApJ, 682, 896

Lang, P., Wuyts, S., Somerville, R. S., et al. 2014, ApJ, 788, 11

Law, D. R., Steidel, C. C., Erb, D. K., et al. 2009, ApJ, 697, 2057

Le Fèvre, O., Saisse, M., Mancini, D., et al. 2003, Proc. SPIE, 4841, 1670

Martig, M., Bournaud, F., Teyssier, R., \& Dekel, A. 2009, ApJ, 707, 250

Maseda, M. V., van der Wel, A., da Cunha, E., et al. 2013, ApJL, 778, L22

McCracken, H. J., Milvang-Jensen, B., Dunlop, J., et al. 2012, A\&A, 544, A156

McNamara, B. R., \& Nulsen, P. E. J. 2007, ARA\&A, 45, 117

Miller, S. H., Bundy, K., Sullivan, M., Ellis, R. S., \& Treu, T. 2011, ApJ, 741,115

Miller, S. H., Ellis, R. S., Sullivan, M., et al. 2012, ApJ, 753, 74

Mortlock, A., Conselice, C. J., Hartley, W. G., et al. 2013, MNRAS, 433, 1185

Muzzin, A., Marchesini, D., Stefanon, M., et al. 2013a, ApJS, 206, 8
Muzzin, A., Marchesini, D., Stefanon, M., et al. 2013b, ApJ, 777, 18 Naab, T., Johansson, P. H., \& Ostriker, J. P. 2009, ApJL, 699, L178

Nesvadba, N. P. H., Lehnert, M. D., De Breuck, C., Gilbert, A. M., \& van Breugel, W. 2008, A\&A, 491, 407

Noeske, K. G., Weiner, B. J., Faber, S. M., et al. 2007, ApJL, 660, L43

Pacifici, C., Charlot, S., Blaizot, J., \& Brinchmann, J. 2012, MNRAS, 421, 2002

Pasquali, A., Gallazzi, A., Fontanot, F., et al. 2010, MNRAS, 407, 937

Peng, C. Y., Ho, L. C., Impey, C. D., \& Rix, H.-W. 2010, AJ, 139, 2097

Puech, M., Flores, H., Hammer, F., et al. 2008, A\&A, 484, 173

Rettura, A., Rosati, P., Strazzullo, V., et al. 2006, A\&A, 458, 717

Rix, H.-W., Guhathakurta, P., Colless, M., \& Ing, K. 1997, MNRAS, 285, 779

Rudnick, G., Rix, H.-W., Franx, M., et al. 2003, ApJ, 599, 847

Schawinski, K., Lintott, C., Thomas, D., et al. 2009, MNRAS, 396, 818

Schiavon, R. P., Faber, S. M., Konidaris, N., et al. 2006, ApJL, 651, L93

Scoville, N., Abraham, R. G., Aussel, H., et al. 2007, ApJS, 172, 38

Scoville, N., Sheth, K., Aussel, H., et al. 2015, arXiv:1511.05149

Shen, S., Mo, H. J., White, S. D. M., et al. 2003, MNRAS, 343, 978

Taylor, E. N., Franx, M., Brinchmann, J., van der Wel, A., \& van Dokkum, P. G. 2010, ApJ, 722, 1

Thomas, D., Maraston, C., Schawinski, K., Sarzi, M., \& Silk, J. 2010 , MNRAS, 404, 1775

Treu, T., Ellis, R. S., Liao, T. X., \& van Dokkum, P. G. 2005, ApJL, 622, L5 Trujillo, I., Förster Schreiber, N. M., Rudnick, G., et al. 2006, ApJ, 650, 18

Tully, R. B., \& Fisher, J. R. 1977, A\&A, 54, 661

van de Sande, J., Kriek, M., Franx, M., Bezanson, R., \& van Dokkum, P. G. 2015, ApJ, 799, 125

van der Wel, A., Bell, E. F., Häussler, B., et al. 2012, ApJS, 203, 24

van der Wel, A., Bell, E. F., van den Bosch, F. C., Gallazzi, A., \& Rix, H.-W. 2009, ApJ, 698, 1232

van der Wel, A., Franx, M., van Dokkum, P. G., et al. 2005, ApJ, 631, 145

van der Wel, A., Franx, M., van Dokkum, P. G., et al. 2014, ApJ, 788, 28

van der Wel, A., Franx, M., Wuyts, S., et al. 2006, ApJ, 652, 97

van der Wel, A., Holden, B. P., Zirm, A. W., et al. 2008, ApJ, 688, 48

van Dokkum, P. G., Franx, M., Kelson, D. D., \& Illingworth, G. D. 1998, ApJL, 504, L17

van Starkenburg, L., van der Werf, P. P., Franx, M., et al. 2008, A\&A, 488, 99 Vogt, N. P., Forbes, D. A., Phillips, A. C., et al. 1996, ApJL, 465, L15

Wake, D. A., van Dokkum, P. G., \& Franx, M. 2012, ApJL, 751, L44 Whitaker, K. E., Franx, M., Bezanson, R., et al. 2015, ApJL, 811, L12 Wild, V., Walcher, C. J., Johansson, P. H., et al. 2009, MNRAS, 395, 144

Worthey, G., Faber, S. M., Gonzalez, J. J., \& Burstein, D. 1994, ApJS, 94, 687 Worthey, G., \& Ottaviani, D. L. 1997, ApJS, 111, 377

York, D. G., Adelman, J., Anderson, J. E., Jr., et al. 2000, AJ, 120, 1579

Ziegler, B. L., Böhm, A., Fricke, K. J., et al. 2002, ApJL, 564, L69 